\title{
Complete single-stage management of left colon cancer obstruction with a new device
}

\author{
J.-H. Kim, ${ }^{1}$ D.-H. Shon, ${ }^{1}$ S.-H. Kang, ${ }^{1}$ B.-I. Jang, ${ }^{2}$ M.-K. Chung, ${ }^{2}$ J.-H. Kim, ${ }^{2}$ M.-C. Shim ${ }^{1}$ \\ ${ }^{1}$ Department of Surgery, College of Medicine, Yeungnam University, 317-1 Daemyungdong Namgu, Daegu, 705-717, Korea \\ 2 Department of Internal Medicine, College of Medicine, Yeungnam University Hospital, 317-1 Daemyungdong Namgu, Daegu, 705-717, Korea \\ Online publication: 14 November 2005
}

[Surg Endosc (2005) 19: 1381-1387, DOI: 10.1007/s00464-004-8232-3]

The authors' affiliations were listed incorrectly in the published version of this article, and the affiliations are corrected here. Correspondence should be addressed to J.-H. Kim at the first affiliation (Department of Surgery). 